\title{
LA PSICOLOGÍA DE SENTIDO COMÚN Y LA TEORÍA DE LA TEORÍA. ALGUNAS REFLEXIONES CRÍTICAS
}

\author{
Eduardo RABOSSI * \\ Universidad de Buenos Aires-CONICET
}

Es bueno comenzar por el principio. En el problema que me ocupa, el principio resulta ser el siguiente. Las decisiones acerca del punto de partida de una investigación no suelen poseer inocencia teórica. Con frecuencia condicionan la índole de los problemas a investigar, determinan el curso de las discusiones y fijan los criterios de pertinencia de las soluciones posibles. La práctica de la Filosofía de la Mente, tal como se desarrolla en el ámbito analítico anglosajón, impone aceptar puntos de partida que se consideran legitimados por la aceptación comunitaria. Uno de ellos consiste en postular la existencia de una psicología de sentido común (folk psychology), atribuirle un carácter teórico, asignarle ciertas entidades y modalidades explicativas propias y discutir sus relaciones con alguna teoría científica de la mente. Este planteo inicial determina las discusiones subsiguientes. Es natural que se discrepe, entonces, acerca de si la psicología de sentido común es reducible a alguna de las teorías científicas preferidas, si es posible eliminarla o vindicarla, si su "constructos" son reales.

En este trabajo me propongo esbozar - sólo esbozar- una argumentación que cuestiona ese punto de partida canónico, ese diseño teórico inicial apa-

He presentado versiones de este trabajo en el Seminario Permanente del Proyecto de Investigación "Modelos de la mente, causación y racionalidad" (Secretaría de Ciencia y Técnica de la Universidad de Buenos Aires, abril 1998) y en el Primer Congreso Iberoamericano de Filosofia (Cáceres/Madrid, septiembre 1998. Mesa redonda: “El desafio de Brentano y la Filosofia de la Mentem, con Olbeth Hansberg, Carlos Moya y Margarita Valdés). Agradezco las observaciones críticas de los miembros del Seminario y de los participantes en la mesa redonda. 
rentemente ineludible. La argumentación forma parte de una tesis más general acerca de la conveniencia de revisar este y otros puntos de partida canónicos de la Filosofía de la Mente. Pienso que una revisión tal puede ayudar a modificar ciertos modos escolásticos de practicarla.

\section{La teoría de la teoría}

En 1970 Paul Churchland proclamó la buena nueva. Al analizar el carácter lógico de las explicaciones de la acción humana, sostuvo,

... el marco conceptual del sentido común en términos del cual nos concebimos a nosotros mismos como personas, tiene todos los rasgos estructurales y lógicos pertinentes de esos marcos conceptuales menores que denominamos teorías científicas.

\section{Y agregó,}

[el marco conceptual del sentido común] contiene un gran número de principios («verdades conceptuales») que son nómicos y no "meramente analíticos».

Churchland no inventó la tesis. Simplemente, puso en blanco y negro ideas sugeridas, desde diferentes puntos de vista, por Dewey, Sellars, Feyerabend, Rorty y Quine. Tuvo éxito. La teoría de que la psicología de sentido común es una teoría pronto se transformó en un dogma compartido por muchos/as filósofos/as de la mente. El dogma sigue en pié.

Hay varias razones que explican la aceptación de la Teoría de la teoría, como se la dió en llamar [Morton. 1980].

En primer lugar, afirmar que la psicología de sentido común es una teoría parece conceder un status claro y decente a algo que no es fácil de ubicar en las categorías y los criterios clasificatorios corrientes.

En segundo lugar, la asignación del un carácter teórico no parece descaminado, al menos de acuerdo a ciertos cánones tradicionales. La lidia cotidiana con nosotros mismos, con otras personas $y$, en general, con el mundo, parece ajustarse a una descripción según la cual explicamos, predecimos, formulamos 
enunciados contrafácticos y hacemos referencia a eventos, estados y procesos, presuponiendo regularidades acerca del comportamiento propio y de los demás y acerca de su relación con los estados mentales pertinentes. Todos estos son, se afirma, los rasgos básicos de una teoría.

En tercer lugar, asignar a la psicología de sentido común un carácter teórico pone un sello de prolijidad en las discusiones sobre su relación con las teorías científicas pertinentes, su posible reducción a ellas y, eventualmente, su eliminación o vindicación final. Ninguna de estas razones es menor.

No es del caso historiar los avatares de la Teoria de la teoría. Baste con señalar que su aceptación no ha producido entre los acólitos la paz conceptual anhelada. La pregunta canónica, ¿̨uál es el contenido y la condición de la psicología de sentido común en tanto teoría y cuál es la índole de su relación con una teoría científica acabada, completa, de la mente?, ha recibido las respuestas más dispares [von Eckardt. 1994; Toribio Mateos. 1995 ].

Quienes piensan que la teoría científica de base va a ser proporcionada por las neurociencias, son propensos a creer que la psicología de sentido común es una teoría muy defectuosa y se sienten tentados a predecir, al menos como una posibilidad abstracta, "que sus principios sean radicalmente falsos y su ontología una ilusión". Consideran entonces la posibilidad de su eventual eliminación y de su substitución por una teoría neurocientífica adecuada [Churchland. 1981].

Los que piensan, en cambio, que la teoría básica será proporcionada por la psicología científica, no coinciden en cuanto al futuro de la psicología de sentido común. Unos tienden a creer que es una teoría defectuosa y concluyen que como es muy difícil que sea vindicada por la psicología científica su suerte final es poco predecible y, quizá, ni siquiera resulte interesante determinarla [Stich. 1983]. Otros consideran que la psicología de sentido común es básicamente una teoría verdadera y que, en consecuencia, las entidades que postula son reales. Por ello tienden a pensar que será vindicada en definitiva por la psicología científica [Fodor. 1975, 1987].

Como se puede apreciar, las discrepancias son muy grandes. No es dificil imaginar a alguien argumentando que en este caso, como en otros, las discrepancias dan la medida del carácter fructífero de una postura filosófica. Cabe replicar a esto que la Teoría de la teoría está afectada por defectos de planteo y de argumentación que hacen dudar de su fecundidad. En lo que sigue expondré varios defectos que considero particularmente graves. 
Los tres defectos básicos de la teoría de la teoría

\section{Los partidarios de la Teoría de la teoría no apelan a un sentido claro y compartido de «teoría»}

Cuando se comparan los planteos de Churchland, Stich, Fodor y Davidson, digamos, respecto del grado de estrictez con que apelan a la noción de teoría, se advierte una especie de degradé que va de la rigurosidad del primero ("Los rasgos estructurales de la psicología de sentido común son perfectamente paralelos a los de la física matemática. La única diferencia descansa en el dominio de las entidade abstractas con las que tratan: números, en el caso de la física, proposiciones en el de la psicología [en esto es única porque no es pitagórica]" [Churchland. 1981]) a la laxa descripción del último. («Aparentemente los pensamientos y decires pertenecen a un modo familiar de explicar el comportamiento y tienen que ser considerados un departamento del sentido común que bien puede ser llamado una teoría. Úna manera de examinar la relación entre el pensamiento y el lenguaje es inspeccionando la teoría implícita en ese tipo de explicación... Prestar atención a las creencias y los deseos para explicar una acción es, en consecuencia, una manera de ubicar una acción en un patrón de comportamiento que la teoría torna coherente" [Davidson. 1975]).

Además, los defensores de la Teoría de la teoría dan por supuesta la legimitidad de una noción de teoría que es cuestionada por muchos filósofos de la ciencia. Churchland, Stich y Fodor, por ejemplo, comparten la misma "Teoría de las teorías científicas". Presuponen que las teorías tienen un carácter proposicional (es decir, están compuestas por ítemes discretos [oraciones, proposiciones] relacionados por mecanismos inferenciales) y que los términos teóricos quedan definidos por el lugar que ocupan en un sistema de leyes. Pero ¿porqué adoptar ese punto de vista? Con razón, Millikan lo ha cuestionado: «la psicología de sentido común no es una teoría rudimentaria... [porque] los ítemes o estados psicológicos no son definidos en base a leyes, ni siquiera a leyes estadísticas". Su cuestionamiento abre un nuevo frente de discusión: «...las categorías de la psicología de sentido común se explican mejor en términos de la teoría evolutiva... la psicología de sentido común es una teoría [que] trata de explicar las acciones postulando cosas o estados internos que tienen ciertas funciones, en vez de cosas que obedecen a leyes». [Millikan 1986]. Es curioso que sólo en tiempos recientes Churchland haya caido en cuenta de que existen 
"serios defectos en las versiones tradicionales de [la Teoría de la teoría al] representar la comprensión de sentido común de la naturaleza humana como un conjunto de oraciones generales internamente almacenadas y las actividades predictivas y explicativas propias como inferencias deductivas a partir de esas oraciones más las premisas ocasionales acerca del caso en cuestión» [Churchland. 1991]. Por supuesto que esta toma de conciencia no es ingenua. Tiene que ver con el cambio de otro de los compromisos asumidos en las primeras versiones de la Teoría de la teoría: el modelo computacional ortodoxo de la mente. Churchland y Stich se han convertido al conexionismo y entonces pretenden dar una justificación diferente a su proclamado cuestionamiento a la psicología de sentido común. La idea es que algunos modelos conexionistas serían incompatibles con los rasgos básicos que, según ellos, caracterizan algunas de las entidades postuladas por la psicología de sentido común. Se argumenta, entonces, que si las hipótesis conexionistas resultaran ser correctas, entonces también lo sería el eliminativismo [Stich et.al. 1991].

En suma, entre los defensores de la Teoría de la teoría no existe acuerdo acerca del sentido preciso en el que se afirma que la psicología de sentido común es, realmente, una teoría.

\section{Ningún defensor de la Teoría de la teoría cuenta y a nadie parece importarle contar con criterios claros de adecuación descriptiva de la psicología de sentido común}

Las discrepancias que acabo de apuntar no sólo se originan en la adopción de enfoques contrapuestos en la Filosofía de la Mente y la Filosofía de las Ciencias, sino en la circunstancia de que la noción en uso de psicología de sentido común sólo permite identificar un "objeto referencial" brumoso, no un "objeto teórico" específico cuya descripción resulte de la aplicación de criterios científicos explicitos. Cuando se pasa revista a las sucesivas caracterizaciones de la psicología de sentido común se advierte que para precisar su género próximo se recurre a expresiones como "marco" ("frame»), "marco estructurado" («framework»), "esquema conceptual» ("conceptual scheme»), "marco estructurado de conceptos" ("framework of concepts"), "red estructurada de principios" («network») y que cuando se intenta identificar la función de la psicología de sentido común aparecen expresiones como "explicar y predecir", "comprender, explicar, predecir y manipular", "explicar y predecir comportamiento característico de la solución de 
problemas", "dar cuenta de las acciones propias y de los demás». Es obvio que esos términos significan cosas muy diversas. Adviértase además la trampa argumentativa que conlleva el empleo de esas expresiones. ¿Qué otra cosa que una teoría puede ser algo que se describe de esa manera?

Hay dos cuestiones adicionales que también quiero señalar. Una es el supuesto de que en el "marco estructurado" que es la psicología de sentido común es posible aislar un tipo de "entidades" peculiares, las actitudes proposicionales, entendidas como los estados de un agente en los que se relaciona con un contenido, una representación, una oración. La otra es la tesis de que en la psicología de sentido común la explicación de la acción de un agente tiene una forma estándar: la asignación de deseos y creencias más la apelación implícita a generalizaciones o leyes; es decir, el silogismo práctico aristotélico resucitado en versión causalista. Según los partidarios de la Teoría de la teoría, las actitudes proposicionales y las explicaciones en términos de deseos y creencias constituyen elementos básicos indubitables de la psicología de sentido común. Sin embargo, cuando se presta atención a su funcionamiento efectivo cabe dudar de la corrección descriptiva de esas postulaciones. Que las actitudes proposicionales existan como entidades reales de la psicología de sentido común con los rasgos que le asignan algunos de los defensores de la Teoría de la teoría (se dice que poseen modularidad proposicional, que son funcionalmente discretas, semánticamente evaluables y causalmente efectivas) es, por supuesto, una cuestión pendiente de decisión hasta contar con una descripción fáctica adecuada. Lo mismo cabe decir de las explicaciones en términos de deseos y creencias. Cuando se dejan a un lado los ejemplos remanidos se advierte que la trama de factores involucrados en las explicaciones corrientes excede notoriamente la simpleza de "explicar la acción de cruzar la calle por parte de Juan apelando a su deseo de fumar y a su creencia de que puede comprar la marquilla en el estanco de la esquina».

3. No hay acuerdo, ni parece haber posibilidad llegar a un acuerdo, acerca de los criterios para decidir a qué tipo de teoría científica corresponde relacionar la psicología de sentido común y cuál es la indole de la relación

Si la psicologia de sentido común es una teoria entonces es posible plantear con sentido un par de preguntas prima facie sencillas: ¿cuál es el ámbito cien- 
tifico especifico con el que dicha teoria debe ser relacionada? ¿cuál es la indole de esa relación? Hacer posible el planteo aparentemente claro de estas preguntas es uno de los atractivos de la Teoría de la teoría. Pero el atractivo se malogra muy pronto. La sencillez y quizá el sentido de las preguntas son sólo rasgos aparentes. En verdad, las respuestas que han generado exhiben discrepancias insalvables. Veamos. Como he señalado al comienzo, hay teóricos de la teoría que refieren la psicologia de sentido común a las neurociencias (en realidad, a una hipotética neurociencia completa), concuerdan en sostener que la relación debe ser reductiva, aunque discrepan en cuanto al carácter eliminativo o conservador de la reducción [los Churchlands]. Por otra parte, quienes refieren la psicología de sentido común a la psicología cientifica, concuerdan en que debe rechazarse la posibilidad de su reducción, pero discrepan en la manera de visualizar el entramado teórico de la disciplina de referencia (una nueva versión del inútil paternalismo teórico de la filosofia vis-a-vis la ciencia). En este respecto, algunos piensan que la psicología cientifica debe ser moldeada por una teoria sintáctica de la mente [Stich], otros que debe serlo por una teoría representacional de la mente plus la hipótesis del lenguaje del pensamiento [Fodor]. Hay quienes refieren la psicología de sentido común a la teoría de la decisión, como un posible módulo de comparación [Davidson]. Estan quienes refieren la psicologia de sentido común a la biologia y piensan que es modelizable en base a ciertos conceptos proveidos por la teoría de la evolución. Un mayor refinamiento expositivo permitiría aumentar el número de discrepancias.

Soy consciente de que lo que he argumentado hasta aquí no tiene un carácter concluyente. He mostrado que los defensores de la Teoría de la teoría usan distintos conceptos de teoría, que varían en la estrictez con que conciben la psicología de sentido común como una teoría, que no precisan qué entienden por psicología de sentido común, que no concuerdan en el tipo de teoría científica con la que cabe relacionarla ni con la índole de la relación. De ello no se sigue, por cierto, que haya probado que la psicología de sentido común no puede tener el carácter teórico que se le atribuye. Lo que sí se sigue -y el punto no es menor- es que el onus probandi acerca de la viabilidad de la Teoría de la teoría ha cambiado: ahora está a cargo de quien decide postularla. Hay, además, otra consecuencia interesante que hace, creo yo, al buen filosofar. Cuando luego de décadas de discusión las dificultades se tornan éndemicas no parece sensato atribuirlas a las posibles limitaciones de las lúcidas mentes filosóficas involucradas, ni al carácter complicado o, aún, misterioso de los problemas. Lo sensato es cargarlas a la cuenta de la tesis de fondo, de los puntos de partida, de las suposiciones. 


\section{¿Es posible una propuesta alternativa?}

Supongamos que he logrado convenceros de que la Teoría de la teoría no es una propuesta adecuada o, al menos, no convincente. Corresponde comenzar de nuevo. Pero, ¿cómo? He aquí una propuesta que creo que merece ser explorada.

Cuando se analiza la polémica estándar acerca de la psicología de sentido común con miras a involucrarse, no por los carriles establecidos, sino en la tarea de identificar la cuestión de fondo en juego, creo que no es dificil descubrir que el problema subyacente (el problema que no se plantea explícitamente y que, en consecuencia, no está resuelto) es el de la condición, la índole o el status del sentido común. En verdad, si «sumamos» la física folk, la biología folk, la psicología folk $\mathrm{y}$, más recientemente, la ética folk, a las que se suele hacer referencia como si fueran campos independientes, lo que tenemos es el área tradicionalmente abarcada con la expresión «sentido común». Si este es el caso, pareciera que tenemos que volver a analizar y discutir un conjunto de problemas cruciales que, por ejemplo, planteó a comienzos de siglo G. E. Moore.

En la dimensión limitada de este trabajo no puedo encarar adecuadamente la cuestión. Lo que sigue es sólo el esbozo muy rudimentario de una posible línea de ataque.

El comienzo de una respuesta interesante pasa por sostener que el género próximo no debe ser el concepto de teoría sino el concepto de práctica. Esto implica que el sentido común - $y$, en consecuencia, la psicología de sentido común- debe ser visto como un complicado saber-hacer, una destreza, habilidad, aptitud o estrategia interpretativa compleja. Para desarrollar esta hipótesis corresponde estudiar detallada y críticamente la extensa bibliografía que antropólogos, psicólogos evolutivos, psicológos sociales, biólogos evolutivos, sociólogos, primatólogos han producido sobre el tema. Ello va a permitir elaborar criterios de adecuación descriptiva y elucidar los mecanismos de adquisición, desarrollo y funcionamiento del sentido común.

El paso del concepto de teoría al concepto de práctica genera, a su vez, varios tipos de oferta filosófica. Me limitaré a los tres más atractivos.

Según el primero, el sentido común involucra una destreza asociada a una ideología subyacente. La destreza es "lo que hemos aprendido a hacer". La ideología es la teoría de la destreza: «lo que nuestras madres y otras personas nos 
han dicho de qué se trataba la cosa cuando enunciaron el saber popular". Corresponde a los filósofos revisar críticamente la ideología y producir una teoría adecuada del funcionamiento efectivo de la destreza. La Postura Intencional, dicen algunos, es una teoría tal. En el caso particular de la psicología de sentido común, este planteo niega la realidad de los estados psicológicos con contenido (deseos y creencias), realza su utilidad instrumental como constructos teóricos de gran poder predictivo y concibe a la destreza como «un cálculo racionalista de interpretación y predicción, un método de interpretación idelizante, abstracto e instrumentalista que ha evolucionado porque funciona y funciona porque ha evolucionado». [Dennett. 1987.1991].

Un segundo tipo de oferta sostiene que la aptitud cognitiva asociada a nuestras transacciones de sentido común, en particular la concerniente a la descripción y explicación de los fenómenos psicológicos, involucra la aptitud de proyectarnos imaginativamente en la perspectiva de otra persona simulando su actividad mental con ayuda de la propia. La llamada "Teoría de la simulación", propuesta por Gordon [1986] y Heal [1986] exhibe un importante desarrollo conceptual que ha culminado en una división interna entre sus cultores ("cartesianos" vs. "conductistas"), motivada por discrepancias acerca de la prioridad a los juicios en primera persona, la existencia de cualidades intrínsecas introspectibles y la prelación respecto de los juicios en tercera persona (Goldman. 1989, 1993 y Gordon. 1992, 1995). Lamentablemente, algunos sostenedores de la Teoría de la simulación admiten la posibilidad de integrar la teoría de la simulación con la Teoría de la teoría (Heal. 1995 y Perner. 1995).

El tercer tipo de oferta propone una estrategia más radical que las anteriores. Consiste en retomar, lisa y llanamente, el tradicional problema del contenido y estatuto del sentido común. Algunos de los rasgos de esta oferta -que es mi preferida-, son los siguientes.

Es importante, ante todo, advertir la complejidad interna de lo que llamamos "sentido común". Es posible postular en él un nucleo duro de convicciones básicas invariables y un conjunto menos duro de opiniones, ideas y conjeturas susceptible a los cambios (alternativamente, puede hablarse del sentido común en un sentido tenue [thin] como distinto de un sentido denso [thick] asociado a épocas o comunidades históricas dadas. Cf. Baker. 1995). Las convicciones básicas son universales y constitutivas de nuestro ser/estar en el mundo. Esas convicciones establecen la naturaleza de las cosas y las personas, hacen posibles las prácticas asociadas y sirven de base a los sistemas de creencias, 
sean ellos laicos o científicos [Rabossi. 1979]. A la pregunta por cuáles son esas convicciones básicas de sentido común, una hipótesis inicial es esta. Son convicciones básicas de sentido común la existencia de objetos macroscópicos, la existencia de personas, la identidad personal, la regularidad de los eventos naturales, y la reactividad emocional hacia objetos y personas. Adviértase que las convicciones básicas de las que hablo no son creencias, opiniones o asentimientos; no involucran transacciones con contenido no tiene sentido hablar de su verdad o falsedad. Tampoco son destrezas o aptitudes. Las convicciones básicas no son conceptualizables en términos de saber /creer ni de saber-hacer, por la sencilla razón que no son saberes.

Puede ofrecerse un argumento trascendental a favor del carácter de las convicciones del sentido común y de su complejidad interna. Alternativamente, se puede ofrecer un argumento que apele a la teoría de la evolución. Los dos tipos de argumentos se complementan.

Esta concepción del sentido común tiene consecuencias filosóficas de monta. En nuestro contexto, las más importantes (algunos dirán, las más graves) son respecto de la ciencia. Si admitimos una concepción fuerte del sentido común, tenemos que negar el

Principio de continuidad: El sentido común y las ciencias conforman un continuo ontológico y cognoscitivo.

Es bueno aclarar que no se niega el Principio de continuidad porque la ciencia no pertenezca al género práctica (sin duda que lo es), sino porque sus presuposiciones, motivaciones, procedimientos y metas, son de una índole distinta de las que estructuran el sentido común. Esto implica romper con la tradición de afirmar, por ejemplo, que la ciencia es el sentido común organizado.

Una concepción fuerte del sentido común también implica negar lo que denominaré el

Principio del imperialismo de la ciencia [versión ontológica]: «La ciencia es la medida de todas las cosas, de las que son en tanto son y de las que no son en tanto no son" [Sellars. 1963].

Negar el Principio del imperialismo de la ciencia implica sostener que hay rasgos básicos de nuestra concepción del mundo que no son susceptibles de ser 
afectados, alterados, cambiados por las teorías científicas. Las convicciones básicas del sentido común son, paradigmáticamente, uno de esos rasgos mundanos. Para apoyar esta hipótesis puede apelarse a una versión ampliada del argumento desarrollado por Strawson respecto del determinismo y la persistencia de las actitudes reactivas [Strawson. 1974].

Cuando se proyecta este planteo a la Filosofía de la Mente se tornan evidentes varias cosas: la irrelevancia de las discusiones acerca de la posible eliminación de la psicología del sentido común, la importancia de reflexionar sobre las implicaciones filosóficas de las convicciones que la constituyen y, sobre todo, la posibilidad de llegar a hablar con fundamento acerca de sus contenidos, complejidad, mecanismos y operatividad.

\section{A modo de conclusión}

Detendré aquí la exposición. He esbozado una línea argumentativa y he señalado mis preferencias. Concluiré con una cita tomada de la entrada autobiográfica de Fodor en el Companion to the Philosophy of Mind. Fodor afirma que "De hecho, el status del supuesto [de que el aparato conceptual de la explicación psicológica folk de sentido común pueda ser adaptado a los requerimientos científicos] se ha transformado en el tópico más importante de la filosofía de la mente». Creo que tiene razón (al menos en esto). Yo pondría las cosas en términos más amplios. De hecho y de derecho el problema de la índole de la psicología de sentido común, de su adecuada descripción y de su relación con las teorías científicas constituye uno de los tópicos más importantes de la Filosofía de la Mente... si dejamos a un lado, por supuesto, el marco inicial de discusión que el canon oficial nos impone.

\section{Referencias bibliográficas}

BAKER, L. (1995) Explaining Attitudes. A Practical Approach to the Mind. Cambridge: CUP.

Churchland, P. M. (1970). "The logical character of action-explanation". Philosophical Review. 79.

- (1981). "Eliminative materialism and the propositional attitudes». Journal of Philosophy. 78. 
- (1991). "Folk psychology and the explanation of human behavior", en J. Greenwood (comp.). The Future of Folk Psychology. Intentionality and Cognitive Science. Cambridge: CUP.

Davidson, D. (1975) «Thought and talk», en D. Davidson. Inquiries Into Truth and Interpretation. Oxford: OUP.

DENNETT, D. (1991). "Two contrasts: Folk crafts versus science and belief versus opinion", en J. Greenwood (comp.). The Future of Folk Psychology. Intentionality and Cognitive Science. Cambridge: CUP.

- Cognitiva. (1989) Vols. 2/3.

ECKARDT, B. von (1994). "Folk Psychology I", en S. Guttenplan (comp.). $A$ Companion to the Philosophy of Mind. Oxford: Blackwell.

FODOR, J. (1975). The Language of Thought. Cambridge, MA.: MIT:

- (1987). Psychosemantics. Cambridge, MA.: MIT.

Greenwood, J. (1991) (comp.) The Future of Folk Psychology. Cambridge: CUP.

GoldMAN, A. (1998). "Interpretation psychologized". Mind and Language. 4.

- (1993). "The psychology of folk psychology». Behavioral and Brain Sciences. 16.

GORDON, R. (1986). "Folk psychology as simulation". Mind and Language. 1.

- (1992). "The simulation theory. Objections and misconceptions». Mind and Language. 7.

- (1995). "Simulation without introspection or inferences from me to you", en T. Stone y M. Davis (comps.) Mental Simulation. Oxford: Blackwell.

HEAL, J. (1986). "Replication and functionalism", en J. Butterfield (comp.) Language, Mind and Logic. Cambridge: CUP.

- (1995). «Simulation, theory, and content», en P. Carruthers y P. Smith (comps.) Theories of theories of Mind. Cambridge: CUP.

MILIKAN, R. (1986) «Biosemantics", Journal of Philosophy. 86.

MORTON, A. (1980). Frames of Mind. Oxford: Clarendon.

PERNER, «Simulation as explicitation of predication-implicit knowledge about the mind: arguments for simulation-theory mix", en P. Carruthers y P. Smith (comps.). Theories of theories of Mind. Cambridge: CUP.

RABOSSI, E. (1979). "¿Porqué el sentido común importa a la filosofía?». Manuscrito. 23.

Ramsay, W., StICH, S. y Garon, J. (1991) "Connectionism, eliminativism and the future of folk psychology", en Greenwood (1991). 
SELlaRs, W. (1956) "Empiricism and the philosophy of mind», en Minnesota Studies in the Philosophy of Science I, Minneapolis: UMP.

- (1963). "Philosophy and the scientific image of man", en W. Sellars. Science, Perception and Reality. Londres: Routledge.

STiCH, S. (1983). From Folk Psychology to Cognitive Science. The Case Against Belief. Cambirdge, MA.: MIT.

STich, S. et. al (1991). "Connectionism, eliminativism, and the future of folk psychology", en J. Greenwood (comp.). The Future of Folk Psychology. Intentionality and Cognitive Science. Cambridge: CUP.

STRAWSON, P. (1974). "Freedom and resentment", en P. Strawson. Freedom and Resentment. Londres: Methuen.

TORibio Mateos, J. (1995) “Eliminativismo y el futuro de la psicología popular», en F. Broncano (comp.). La mente humana. Madrid: Trotta.

WILKES, K. (1984) «Pragmatics in science and theory in common sense». Inquiry. 27. WitTGENSTEIn, L. (1969) Über Gewissheit. Oxford. Blackwell. 